(2) Open Access Full Text Article

REVIEW

\title{
Potential of ponatinib to treat chronic myeloid leukemia and acute lymphoblastic leukemia
}

This article was published in the following Dove Press journal:

OncoTargets and Therapy

19 August 2013

Number of times this article has been viewed

\section{Kimberly E Price \\ Najma Saleem \\ Georgina Lee \\ Michael Steinberg}

Massachusetts College of Pharmacy and Health Sciences University, Worcester, MA, USA
Correspondence: Michael Steinberg Massachusetts College of Pharmacy and Health Sciences University, 19 Foster Street, Worcester, MA 01608, USA

Tel +I 5083735666

Email michael.steinberg@mcphs.edu

\begin{abstract}
Development of BCR-ABL tyrosine kinase inhibitors (TKIs) have improved outcomes for patients diagnosed with chronic myeloid leukemia and Philadelphia chromosome positive acute lymphoblastic leukemia. However, resistance or intolerance to these TKIs still leaves some patients without many treatment options. One point mutation in particular, the T315I mutation, has been shown to be resistant to first and second generation TKIs. The third generation TKI, ponatinib, may provide an option for these patients. Ponatinib (Iclusig ${ }^{\circledR}$ ), an orally available, pan-tyrosine kinase inhibitor has a unique binding mechanism allowing inhibition of BCR-ABL kinases, including those with the T315I point mutation. A Phase II study evaluated ponatinib in patients who were resistant or intolerant to nilotinib or dasatinib or patients who had the T315I mutation. In the Phase II study, ponatinib produced a major cytogenetic response in 54\% of chronic phase chronic myeloid leukemia patients. It further achieved major hematologic response in $52 \%$ of patients in the accelerated phase, $31 \%$ of patients in the blast phase, and $41 \%$ of Philadelphia chromosome positive acute lymphoblastic leukemia patients. Ponatinib also showed efficacy in patients with the T315I mutation. Serious adverse events included arterial thrombosis, hepatotoxicity, cardiovascular risks, pancreatitis, hemorrhage, fluid retention, myelosuppression, rash, abdominal pain, and embryo-fetal toxicity. Due to the risk of these adverse events and potential drug interactions, the use of ponatinib must be carefully weighed against the benefits in treating patients who have limited treatment options.
\end{abstract}

Keywords: BCR-ABL, tyrosine kinase inhibitor, TKI, T315I, Philadelphia chromosome

\section{Introduction}

The development and advancement of tyrosine kinase inhibitors (TKIs) has contributed to improved outcomes in patients with Philadelphia chromosome positive $(\mathrm{Ph}+)$ chronic myeloid leukemia (CML) and acute lymphoblastic leukemia (ALL). The development of imatinib, the first commercially available TKI in 2001, revolutionized the treatment of CML and Ph+ ALL by targeting the BCR-ABL protein which is responsible for many of the pathologic processes of these malignancies. By eliminating the oncogenic activity of the BCR-ABL enzyme, imatinib is able to inhibit proliferation and induce apoptosis in BCR-ABL positive CML and $\mathrm{Ph}+$ ALL cells. ${ }^{2}$ However, some patients treated with imatinib do not achieve the desired complete cytogenetic response (CCyR), and those who do may relapse. More potent second-generation TKIs, such as dasatinib and nilotinib, were developed to improve upon those responses observed with imatinib. In addition to BCR-ABL, dasatinib inhibits other oncogenic kinases including the Src family kinases, c-Kit, and plateletderived growth factor receptors (PDGFR). ${ }^{3}$ Nilotinib inhibits kinase targets includ- 
ing BCR-ABL, PDGFR, and c-Kit, but not the Src family kinases. ${ }^{4}$ While the first and second-generation TKIs have demonstrated improved outcomes in patients with CML and $\mathrm{Ph}+\mathrm{ALL}$, resistance still occurs in a subset of patients who harbor BCR-ABL mutations, particularly the T315I mutant. Until the recent approval of ponatinib (Iclusig ${ }^{\circledR}$, ARIAD Pharmaceuticals, Cambridge, MA, USA), there were no approved therapies for patients who had developed resistance, refractoriness, or intolerance to commercially available TKIs. Ponatinib has a unique mechanism of action and binding location on the BCR-ABL kinase that has been shown to overcome observed resistance to other TKIs and offers additional promise to patients with $\mathrm{Ph}+$ CML and ALL.

\section{Chronic myeloid leukemia}

CML is a hematologic stem cell neoplasm characterized by unregulated growth of myeloid cells in the bone marrow. It affects approximately 1-2 per 100,000 adults, with approximately 5,000 new cases diagnosed annually in the United States. ${ }^{1,5}$ Incidence of CML has nearly doubled since 2001, and this trend is anticipated to continue. Of note is the increase in the duration of survival secondary to a reduction in all-cause mortality of patients with CML due to improved management of the disease with TKIs. This success is expected to plateau at approximately 181,000 patients living with CML by $2050{ }^{1}$ Most patients with CML are diagnosed in the chronic phase of the disease, in which symptoms are mild; however, if left untreated, the disease is likely to progress to the accelerated phase, and finally the blast phase, for which currently available treatments are typically least effective. ${ }^{6}$

In CML patients, the $A B L$ gene on chromosome 9 undergoes a reciprocal translocation with the $B C R$ gene on chromosome 22, creating what is commonly known as the Philadelphia chromosome, which is expressed as a tyrosine kinase. Treatment of CML includes stem cell transplant, chemotherapy, and targeted therapy with TKIs. There are currently five TKIs approved for the treatment of CML: the first generation TKI, imatinib; second generation TKIs, dasatinib, nilotinib, and bosutinib; and the third generation TKI, ponatinib. ${ }^{2-4,7,8}$

\section{Acute lymphoblastic leukemia}

ALL is a cancer of the blood and bone marrow in which the body produces excessive lymphoblasts which do not mature into normal functioning lymphocytes. ALL is the most common type of cancer in children but is also seen in adults. ${ }^{9}$ More than $95 \%$ of children with ALL achieve remission with treatment, and $80 \%$ are expected to have a long life span without a relapse. ${ }^{9}$ Unfortunately, the overall prognosis in ALL patients over 25-35 years of age is not as positive as in children. ${ }^{10,11}$ A confounding factor for adult patients is the presence of the Philadelphia chromosome, which is present in approximately $30 \%$ of adult ALL patients and likely imparts upon these individuals a poorer prognosis than those without this chromosomal abnormality. ${ }^{10,12}$ Less than $5 \%$ of children with ALL are $\mathrm{Ph}+{ }^{13}$

Due to rapid progression of the disease, treatment should be started as soon as possible following diagnosis. One treatment option for patients includes treatment with TKIs. TKIs can be used in patients who are $\mathrm{Ph}+$ and are newly diagnosed or have relapsed or have refractory ALL. In pediatric patients, imatinib is the only approved TKI for ALL treatment, while imatinib, dasatinib, and ponatinib have been evaluated and approved for use in adult patients.

\section{Ponatinib mechanism of action}

Ponatinib is an orally administered, synthetic, multi-targeting TKI structurally designed as a pan-BCR-ABL inhibitor. This is reflected by the inhibition of in vitro tyrosine kinase activity of ABL and T315I mutant ABL with a half maximal inhibitory concentration $\left(\mathrm{IC}_{50}\right)$ of $0.4 \mathrm{nM}$ and $2.0 \mathrm{nM}$, respectively. ${ }^{8}$ Ponatinib also inhibits the in vitro activity of additional oncogenic kinases with $\mathrm{IC}_{50}$ between $0.1 \mathrm{nM}$ and $20 \mathrm{nM}$, including members of the vascular endothelial growth factor receptor (VEGFR), PDGFR, fibroblast growth factor receptor (FGFR), ephrin receptor, Src family kinases, c-Kit, RET, TIE2, and FLT3. ${ }^{8}$ These additional oncogenic kinases have been implicated in the pathogenesis of acute myeloid leukemia as well, which furthers the importance of a panBCR-ABL inhibitor as a treatment option.

The ponatinib molecule is designed to interact with T315I in a different manner than the existing kinase inhibitors. Most ABL inhibitors can be classified as either a DFG-in (type 1 inhibitor) or DFG-out (type 2 inhibitor) compound, depending on their binding interactions with BCR-ABL. Type 1 inhibitors, or DFG-in compounds, are considered the first generation of small molecule kinase inhibitors targeting the ATP-binding site of the enzyme in the active form, which is characterized by an open conformation of the activation loop. ${ }^{14}$ In contrast, type 2 inhibitors, or DFG-out compounds, are the inactive forms subsequently developed to bind to an additional hydrophobic site in addition to the same area as type 1 compounds. ${ }^{14}$ The ability to bind to an additional hydrophobic site provides type 2 inhibitors with the ability to be more selective and more potent than 
type 1 inhibitors. ${ }^{14}$ Examples of DFG-in inhibitors include sunitinib and dasatinib, while DFG-out inhibitors include imatinib, nilotinib, and ponatinib. Regardless of binding mode, imatinib, dasatinib, and nilotinib form a key hydrogen bond at the side chain of the gatekeeper residue Thr315 in native $\mathrm{ABL}$ and also contain a lipophilic moiety that binds to a hydrophobic pocket located near Thr315. ${ }^{15}$ However, this key hydrogen bond is compromised when the gatekeeper residue mutates to isoleucine, which can also cause a steric clash with the inhibitor and block entry to the hydrophobic pocket adjacent to Thr315. ${ }^{15}$ As such, imatinib, dasatinib, and nilotinib are unable to block the ABL kinase activity of Thr315 point mutations. This highly resistant variation present in the gatekeeper region of the ATP-binding site in the ABL kinase domain of BCR-ABL fusion protein accounts for $15 \%-20 \%$ of clinically observed mutations. ${ }^{15}$ In contrast, ponatinib was designed to avoid binding with the side chain of T315I in native BCR-ABL, and instead forms beneficial van der Waals interactions with the isoleucine side chain of the T315I mutant. ${ }^{16}$ This, coupled with its ability to accommodate the steric hindrance from mutations in the isoleucine variant, allows it to bind to and inhibit the ABL kinase activity of T315I point mutations.

\section{Methods}

Literature searches were conducted on March 18, 2013 using Ovid MEDLINE ${ }^{\circledR}$, from 1946 to March, week 1, 2013 and using the Embase biomedical database, from 1980 to week 11, 2013. The following search terms were included and were organized in the following format: ponatinib or Iclusig, and acute lymphoblastic leukemia or chronic myeloid leukemia or chronic myelogenous leukemia. The search, which was limited to the English language and to clinical trials conducted in humans, produced nine results. The results of clinical trials and safety information were obtained from the manufacturer's prescribing information and the US Food and Drug Administration review of ponatinib. All identified publications are included in this discussion.

\section{Pharmacology}

The chemical name for ponatinib hydrochloride is 3-(2-imidazo[1,2-b]pyridazin-3-ylethynl)-4-methyl-N-[4[(4-methylpiperazin-1-yl)methyl]-3-(trifluoromethyl)phenyl] benzamide hydrochloride, and its molecular formula is $\mathrm{C}_{29} \mathrm{H}_{28} \mathrm{CIF}_{3} \mathrm{~N}_{6} \mathrm{O}$, with a formula weight of $569.02 \mathrm{~g} / \mathrm{mol}^{8}{ }^{8}$ Ponatinib is an off-white to yellow powder with a $\mathrm{pKa}$ of 2.77 and 7.8, and its solubility is inversely proportional to its $\mathrm{pH} .{ }^{8}$ The tablets are available as white, round, and film-coated for oral administration, and each tablet contains $15 \mathrm{mg}$ or $45 \mathrm{mg}$ ponatinib.

\section{Pharmacokinetics}

The mean peak concentration and area under the curve of ponatinib $45 \mathrm{mg}$ daily at steady state in patients with advanced hematologic malignancies are $73 \mathrm{ng} / \mathrm{mL}$ and $1,253 \mathrm{ng} \cdot \mathrm{hour} / \mathrm{mL}$, respectively. Peak concentrations of ponatinib were observed within 6 hours of oral administration. High-fat or low-fat meals have not been observed to have an effect on ponatinib plasma concentrations. ${ }^{8}$ Ponatinib is greater than $99 \%$ bound to plasma proteins in vitro, and it is a weak substrate for both P-glycoprotein and ATPbinding cassette sub-family $\mathrm{G}$ member 2 (also known as breast cancer resistance protein) in vitro. At least $64 \%$ of a ponatinib dose undergoes phase I and phase II metabolism. CYP3A4, and to a lesser extent CYP2C8, CYP2D6, and CYP3A5, are involved in phase I metabolism of ponatinib in vitro. Ponatinib is mainly eliminated via feces and has an elimination half-life of approximately 24 hours. Hepatic elimination is another major route of excretion, yet ponatinib has not been studied in patients with hepatic or renal impairment. ${ }^{8}$ Due to involvement by the liver in metabolism, hepatic impairment may result in increased ponatinib concentrations, which could potentially lead to an increase of adverse events. ${ }^{8}$

\section{Pharmacodynamics}

In the Phase I trial by Cortes et al, an overall assessment of the pharmacodynamic response in each patient was based on a comparison of average phosphorylated CrkL ( $p$ CrkL) levels at posttreatment trough time points compared to $\mathrm{p}$ CrkL levels at baseline. ${ }^{17}$ Phosphorylated CrkL, a BCR-ABL adaptor protein, serves as a surrogate of BCR-ABL activity in vivo and has been recognized as a prognostic parameter in patients with leukemia treated with tyrosine kinase inhibitors, including ponatinib.

A total of $43 \mathrm{Ph}+$ patients were evaluated for pharmacodynamic assessment and a reduction of $\mathrm{p}$ CrkL levels. In patients receiving $8 \mathrm{mg}$ of ponatinib, a reduction of $50 \%$ or more in CrkL phosphorylation was observed in four of six patients $(67 \%)$. At doses of $15 \mathrm{mg}$ or more, 32 of 34 patients (94\%) had a reduction of $50 \%$ or more in p CrkL, and this included eight of ten patients (80\%) with the T315I mutation. ${ }^{17}$ This trial provided valuable evidence of ponatinib's direct kinase inhibiting activity in patients with a variety of BCR-ABL mutations, including the T315I mutant, leading to further evaluation of its effects. 


\section{Preclinical}

Ponatinib has been observed to achieve marked reductions in levels of phosphorylated BCR-ABL and CrkL within 6 hours of administration in a mouse model injected with T315Ivariant $\mathrm{BCR}-\mathrm{ABL}$ expressing $\mathrm{Ba} / \mathrm{F} 3$ cells. ${ }^{16}$ Additional dose-dependent effects such as tumor regression and mean tumor volume reduction were observed following oral ponatinib administration in mice xenografted with T315I-variant BCR-ABL-expressing Ba/F3 cells. ${ }^{16}$ However, this effect has not been evaluated in humans. Other preclinical data have replicated these results and support outcomes consistent with prolonged survival and tumor regression in similarly prepared mice.

Analogous results were also observed when ponatinib was administered in mice xenografted with other oncogenic BCR-ABL mutant cell types. In a study evaluating ponatinib ( $1 \mathrm{mg} / \mathrm{kg}$ to $25 \mathrm{mg} / \mathrm{kg}$ for 28 days) in mice xenografted with MV4-11 cells, $46 \%$ inhibition of tumor growth was seen at the $1 \mathrm{mg} / \mathrm{kg}$ dose, with tumor regression seen at doses $\geq 2.5 \mathrm{mg} / \mathrm{kg}$. ${ }^{16}$ Following a 31 -day follow-up period, no palpable tumors were present in mice who received $10 \mathrm{mg} / \mathrm{kg}$ and $25 \mathrm{mg} / \mathrm{kg}$ of ponatinib. ${ }^{16}$ The effects of ponatinib $(30 \mathrm{mg} / \mathrm{kg} /$ day) were also tested in mice with FGFRmutated endometrial cancer and FGFR3-mutated bladder cancer xenografts, which revealed $80 \%$ reduction in tumor growth compared with controls. ${ }^{16}$ Ponatinib $(10 \mathrm{mg} / \mathrm{kg} / \mathrm{day})$ also induced a $56 \%$ tumor regression in a mouse xenograft model of FGFR2-upregulated gastric cancer. ${ }^{16}$

Preclinical data suggested that plasma levels higher than the in vitro $\mathrm{IC}_{50}$ values obtained for BCR-ABL mutants could be maintained for $>6$ hours. Ponatinib inhibited native ABL with an $\mathrm{IC}_{50}$ of $0.37 \mathrm{nM}$ and clinically relevant BCR-ABL mutations T315I, G252H, T253P, M351T, and H396A with
$\mathrm{IC}_{50}$ values of $2.0 \mathrm{nM}, 0.44 \mathrm{nM}, 0.30 \mathrm{nM}, 0.30 \mathrm{nM}$, and $0.35 \mathrm{nM}$, respectively. ${ }^{16}$ In addition, ponatinib inhibited the Src family, VEGFR, FGFR, PDGFR, and c-Kit with $\mathrm{IC}_{50}$ values of $5.4 \mathrm{nM}, 1.5 \mathrm{nM}, 2.2 \mathrm{nM}, 1.1 \mathrm{nM}$, and $12.5 \mathrm{nM}$, respectively. ${ }^{16}$ In summary, preclinical data supports the conclusion that ponatinib is an orally active, pan-TKI that inhibits native $\mathrm{BCR}-\mathrm{ABL}$ and most $\mathrm{BCR}-\mathrm{ABL}$ mutants, including the clinically relevant T315I mutant, with high potency.

\section{Reproductive toxicity, mutagenicity, carcinogenicity}

Fertility studies using ponatinib have not been published. However, findings from toxicology studies reveal that ponatinib may impair male and female fertility, as epithelial degeneration in both rat and simian testes has been observed, along with follicular atresia with associated endometrial atrophy in ovaries of monkeys. ${ }^{8}$ This has resulted in the designation of ponatinib as a pregnancy category $\mathrm{D}$ drug. Ames assays have not indicated any degree of mutagenicity following bacterial exposure to ponatinib, while the potential for carcinogenicity of ponatinib is not known. ${ }^{8}$

\section{Clinical studies Phase I}

In a Phase I dose escalation study by Cortes et al, 81 patients with resistant hematologic cancers, including 60 patients with CML and five patients with $\mathrm{Ph}+\mathrm{ALL}$, were administered ponatinib orally once daily at one of seven dose levels ( $2 \mathrm{mg}$, $4 \mathrm{mg}, 8 \mathrm{mg}, 15 \mathrm{mg}, 30 \mathrm{mg}, 45 \mathrm{mg}$, and $60 \mathrm{mg}) .{ }^{17}$ Baseline characteristics of the patients are listed in Table 1. Doselimiting toxic effects observed in treated patients included elevated lipase $(12 / 81 ; 15 \%)$ or amylase levels $(3 / 81 ; 4 \%)$ and progression to pancreatitis $(11 / 81 ; 14 \%)$. The deter-

Table I Clinical characteristics of patients at baseline

\begin{tabular}{|c|c|c|c|c|}
\hline & $\begin{array}{l}\text { Chronic phase CML } \\
(n=43)\end{array}$ & $\begin{array}{l}\text { Accelerated phase CML } \\
(n=9)\end{array}$ & $\begin{array}{l}\text { Blast phase CML } \\
(n=8)\end{array}$ & $\begin{array}{l}\text { Ph-positive ALL } \\
(n=5)\end{array}$ \\
\hline Age in years (range) & $55(27-85)$ & $6 \mathrm{I}(42-77)$ & $5 \mathrm{I}(26-73)$ & $36(27-67)$ \\
\hline \multicolumn{5}{|l|}{ Sex } \\
\hline Male & $21(49 \%)$ & $6(67 \%)$ & $5(62 \%)$ & $5(100 \%)$ \\
\hline Female & $22(51 \%)$ & $3(33 \%)$ & $3(38 \%)$ & 0 \\
\hline $\begin{array}{l}\text { Median time from diagnosis to treatment } \\
\text { years (range) }\end{array}$ & $6.6(0.8-23.5)$ & $6.7(2.7-16.2)$ & $6.5(1.6-19.8)$ & $1.2(0.8-1.9)$ \\
\hline \multicolumn{5}{|l|}{ Hematologic analysis (values are $\times 10^{-3} / \mathrm{mm}^{3}$ ) } \\
\hline Median white-cell count (range) & $5.8(0.6-65.2)$ & $27.9(1.6-2 \mid 2.7)$ & $9.5(1.5-36.9)$ & $14.9(0.2-92.7)$ \\
\hline Median platelet count (range) & $217.5(28-900)$ & $224.0(28-1400)$ & $22.0(13-172)$ & $\mid 4.0(7-7 \mid)$ \\
\hline \multicolumn{5}{|l|}{ Previous use of tyrosine kinase inhibitors } \\
\hline$\geq 2$ drugs & $42(98 \%)$ & $9(100 \%)$ & $8(100 \%)$ & $2(40 \%)$ \\
\hline$\geq 3$ drugs & 27 (63\%) & $8(89 \%)$ & $6(75 \%)$ & 0 \\
\hline
\end{tabular}

Abbreviations: CML, chronic myeloid leukemia; ALL, acute lymphoblastic leukemia. 
mined maximum tolerated dose was $45 \mathrm{mg}$, which was later established as the approved dose for treatment. Efficacy endpoints included major cytogenetic response (MCyR), which included partial cytogenic response and CCyR, complete hematologic response, major hematologic response (MaHR), and major molecular response (MMR). Efficacy data from the Phase I trial is shown in Table 2.

\section{Phase II}

The Phase II PACE (ponatinib Ph+ ALL and CML evaluation) study was an international, open label, single-arm, multi-center trial that included patients with chronic phase CML (CP-CML), accelerated phase CML (AP-CML), blast phase CML (BP-CML), or Ph+ ALL, in which patients were divided into treatment cohorts based on their disease presentation. ${ }^{8,18}$ Patients were documented as either resistant or intolerant to dasatinib or nilotinib or positive for the T315I mutation. A total of 449 patients were enrolled to receive $45 \mathrm{mg}$ ponatinib orally once daily. Five patients were excluded from the efficacy analysis as they lacked the T315I mutation and did not have prior dasatinib or nilotinib use. Of the 444 patients included in the efficacy analysis, 267 were diagnosed with CP-CML, 83 with APCML, 62 with BP-CML, and 32 were diagnosed with $\mathrm{Ph}+$ ALL. A total of 128 patients were positive for the T315I mutation.

The primary endpoint for CP-CML patients was MCyR (ie, CCyR plus partial cytogenic response). Secondary endpoints included complete hematologic response and MMR.

AP- and BP-CML patients and Ph+ ALL patients were evaluated for MaHR, which was defined as either a complete hematologic response or no evidence of leukemia. Secondary endpoints in these patients were CCyR, partial cytogenic response, MCyR, and MMR. These endpoints are consistent with those used in Phase III studies of dasatinib and nilotinib in resistant or intolerant CML or Ph+ ALL patients.
At the time of analysis, 144 of $267 \mathrm{CP}-\mathrm{CML}$ patients (54\%) achieved a MCyR. Of the 83 AP-CML patients, 43 (52\%) achieved MaHR, while 19 of 62 (31\%) BP-CML and 13 of $32(41 \%) \mathrm{Ph}+\mathrm{ALL}$ patients achieved MaHR overall (see Table 3). These results support the use of ponatinib in patients in any phase of CML, as well as Ph+ ALL patients, who are either intolerant or resistant to dasatinib or nilotinib.

\section{Phase III}

The Phase III EPIC (evaluation of ponatinib versus imatinib in chronic myeloid leukemia) study is currently exploring the safety and efficacy of ponatinib compared to imatinib at 175 sites across North America, Europe, and Asia-Pacific. ${ }^{19}$ Anticipated enrollment is approximately 500 newly diagnosed (within 6 months) patients with CP-CML, randomized 1:1 to either ponatinib $45 \mathrm{mg}$ once daily or imatinib $400 \mathrm{mg}$ once daily. The primary endpoint is achievement of MMR at 12 months. Additional study outcomes include rate of MMR at 5 years, molecular response at 3 months, CCyR at 12 months, progression-free survival, and overall survival. Patients will be followed for up to 8 years to determine long term survival rates.

\section{Safety of ponatinib}

In the Phase I trial, the most common side effects were skin disorders and constitutional symptoms. Skin disorders included rash, acneiform dermatitis, and dry skin. Rash was the most common adverse event reported $(n=26$; $32 \%)$. Constitutional symptoms included arthralgia $(\mathrm{n}=14$; $17 \%)$, fatigue $(\mathrm{n}=11 ; 14 \%)$, and nausea $(\mathrm{n}=11 ; 14 \%)$. Other common adverse events with a reported incidence of $\geq 10 \%$ included increased lipase ( $\mathrm{n}=12 ; 15 \%$ ), headache $(n=10 ; 12 \%)$, hypertriglyceridemia $(n=10 ; 12 \%)$, myalgia $(n=10 ; 12 \%)$, increased liver transaminases, with an increased alanine aminotransferase $(\mathrm{n}=8 ; 10 \%)$ and increased aspartate aminotransferase $(n=7 ; 9 \%) .{ }^{17}$

Table 2 Response from ponatinib treatment: Phase I trial data

\begin{tabular}{|c|c|c|c|c|}
\hline & \multicolumn{2}{|c|}{$\begin{array}{l}\text { Chronic phase CML } \\
(n=43)\end{array}$} & \multicolumn{2}{|c|}{$\begin{array}{l}\text { Accelerated phase CML, blast phase CML, and Ph+ ALL } \\
(n=22)\end{array}$} \\
\hline & $\begin{array}{l}\text { All patients } \\
(n=43)\end{array}$ & $\begin{array}{l}\text { T3 I5 I mutation } \\
(n=12)\end{array}$ & $\begin{array}{l}\text { All patients } \\
(n=22)\end{array}$ & $\begin{array}{l}\text { T3 I I I mutation } \\
(n=7)\end{array}$ \\
\hline CCyR & $27(63 \%)$ & $9(75 \%)$ & $3(14 \%)$ & I (I4\%) \\
\hline MCyR & 31 (72\%) & II (92\%) & 7 (32\%) & $2(29 \%)$ \\
\hline $\mathrm{CHR}$ & 42 (98\%) & 12 (I00\%) & $\mathrm{N} / \mathrm{A}$ & N/A \\
\hline $\mathrm{MaHR}$ & N/A & N/A & $8(36 \%)$ & 2 (29\%) \\
\hline MMR & 19 (44\%) & 8 (67\%) & $2(9 \%)$ & 2 (29\%) \\
\hline
\end{tabular}

Abbreviations: CCyR, complete cytogenetic response; CHR, complete hematologic response; CML, chronic myeloid leukemia; MaHR, major hematologic response; MCyR, major cytogenetic response; MMR, major molecular response; N/A, not available; Ph+ ALL, Philadelphia chromosome positive acute lymphoblastic leukemia. 
Table 3 Response rates from PACE (ponatinib Ph+ ALL and CML evaluation)

\begin{tabular}{|c|c|c|c|}
\hline CP-CML & Overall $(n=267)$ & Resistant/intolerant $(n=203)$ & $T 3|5|(n=64)$ \\
\hline MCyR \% (95\% Cl) & $54 \%(48-60)$ & $49 \%(42-56)$ & $70 \%(58-8 I)$ \\
\hline Median time to MCyR (range) & 84 days (49-334 days) & $\mathrm{N} / \mathrm{A}$ & $N / A$ \\
\hline CCyR \% $(95 \%$ Cl) & $44 \%(38-50)$ & $37 \%(31-44)$ & $66 \%(52-77)$ \\
\hline AP-CML & Overall $(n=83)$ & Resistant/intolerant $(n=65)$ & $T 3|5|(n=\mid 8)$ \\
\hline $\mathrm{MaHR} \%(95 \% \mathrm{Cl})$ & $52 \%(4 I-63)$ & $55 \%(43-68)$ & $39 \%(17-64)$ \\
\hline Median time to MaHR (range) & 21 days $(12-176$ days $)$ & $N / A$ & $N / A$ \\
\hline Median duration of MaHR (range) & 9.5 months (I.I-I7.7 months) & $N / A$ & $N / A$ \\
\hline $\mathrm{CHR} \%(95 \% \mathrm{Cl})$ & $44 \%(33-55)$ & $45 \%(32-57)$ & $39 \%(17-64)$ \\
\hline BP-CML & Overall $(n=62)$ & Resistant/intolerant $(n=38)$ & $T 3|5|(n=24)$ \\
\hline MaHR \% (95\% Cl) & $31 \%(20-44)$ & $32 \%(18-42)$ & $29 \%(|3-5|)$ \\
\hline Median time to MaHR (range) & 29 (12-II3 days) & $N / A$ & $N / A$ \\
\hline Median duration of MaHR (range) & 4.7 months (I.8-14.I+ months) & $\mathrm{N} / \mathrm{A}$ & $N / A$ \\
\hline CHR \% $(95 \% \mathrm{Cl})$ & $21 \%(12-33)$ & $24 \%(1 I-40)$ & $17 \%(5-37)$ \\
\hline$P h+A L L$ & Overall $(n=32)$ & Resistant/intolerant $(n=10)$ & $T 3|5|(n=22)$ \\
\hline $\mathrm{MaHR} \%(95 \% \mathrm{Cl})$ & $41 \%(24-59)$ & $50 \%(19-81)$ & $36 \%(17-59)$ \\
\hline Median time to MaHR (range) & 20 ( $11-168$ days $)$ & $\mathrm{N} / \mathrm{A}$ & $N / A$ \\
\hline Median duration of MaHR (range) & 3.2 months (I.8-8.8+ months) & $N / A$ & $N / A$ \\
\hline CHR \% (95\% Cl) & $34 \%(19-53)$ & $40 \%(12-74)$ & $32 \%(14-55)$ \\
\hline
\end{tabular}

Abbreviations: AP-CML, accelerated phase CML; BP-CML, blast phase CML; CCyR, complete cytogenetic response; CHR, complete hematologic response; Cl, confidence interval; CML, chronic myeloid leukemia; MaHR, major hematologic response; MCyR, major cytogenetic response; Ph+ ALL, Philadelphia chromosome positive acute lymphoblastic leukemia; CP, chronic phase.

Serious adverse events seen in the Phase I trial involved pancreatitis and myelosuppression. Pancreatitis occurred in eleven patients (14\%) and was serious in eight patients $(10 \%)$.

Adverse events of grade 3 or higher were considered serious, and grading was in accordance with the National Cancer Institute's Common Terminology Criteria for Adverse Events, version 3.0. ${ }^{20}$ Elevated levels of lipase or amylase without pancreatitis occurred in an additional seven patients (9\%). Interestingly, the incidence of pancreatitis or elevated serum lipase/amylase concentrations occurred less frequently as the dose of ponatinib increased. Time to onset of pancreatitis varied with the increase in dose. The median time to first event of pancreatitis, elevated lipase, or elevated amylase was 22 days at a dose of $30 \mathrm{mg}$ in five patients out of seven (71\%), 40 days at a dose of $45 \mathrm{mg}$ in eight patients out of $31(36 \%)$, and 6.5 days at a dose of $60 \mathrm{mg}$ in four out of 19 patients (21\%). The pancreatitis resolved within a median of 6 days. Rechallenge was successful in most patients, and only two patients permanently discontinued ponatinib as a result of pancreatitis. Treatment-related myelosuppression was also common. Twenty-seven percent ( 22 of 81 total) of patients experienced thrombocytopenia, $12 \%(\mathrm{n}=10)$ experienced neutropenia, and $10 \%(\mathrm{n}=8)$ experienced anemia. Lymphopenia and leucopenia were also seen. A complicating factor in the assessment of myelosuppression was the observation of reduced blood cells at baseline, particularly in patients with AP-CML, BP-CML, and Ph+ALL. ${ }^{8}$

In the Phase II trial, the most common $(\geq 20 \%)$ nonhematologic adverse reactions were hypertension $(\mathrm{n}=300$; $67 \%)$, rash $(\mathrm{n}=175 ; 39 \%)$, abdominal pain $(\mathrm{n}=167 ; 37 \%)$, fatigue $(\mathrm{n}=141 ; 31 \%)$, headache $(\mathrm{n}=123 ; 27 \%)$, dry skin $(n=115 ; 26 \%)$, constipation $(n=134 ; 30 \%)$, arthralgia $(n=89$; $20 \%)$, nausea $(\mathrm{n}=104 ; 23 \%)$, and pyrexia $(\mathrm{n}=111 ; 25 \%)$. These events were similar to what was seen in the Phase I trial. Hematologic adverse reactions included thrombocytopenia, anemia, neutropenia, lymphopenia, and leukopenia. ${ }^{8}$

Serious adverse events seen in the PACE trial included arterial thrombosis and hepatotoxicity. Eleven percent (51/449 total) of ponatinib treated patients experienced arterial thrombosis, and the event was serious in $8 \%(n=34)$ of patients. Myocardial infarction or worsening coronary artery disease $(n=21 ; 5 \%)$ was the most common arterial thrombosis event. Of the patients who experienced a serious arterial thrombosis event, $62 \%(n=21)$ required a revascularization procedure. Fatal cases of acute liver failure were also seen. Fulminant hepatic failure occurred in one patient, who died within one week of starting ponatinib. The incidence of serum alanine aminotransferase and aspartate aminotransferase elevations were $56 \%$ for all grades, and $8 \%$ for grades 3 or $4 .{ }^{8}$ As a result, both arterial thrombosis and hepatotoxicity have been included in boxed warnings in the manufacturer's prescribing information. 
The PACE trial also revealed issues of congestive heart failure, fluid retention, cardiac arrhythmias, and hypertension. Congestive heart failure occurred in 33 patients $(7 \%)$ and was considered serious in 27 patients (4\%). Fluid retention occurred in $23 \%$ of patients, and the most commonly observed events were peripheral edema (16\%), pleural effusion (7\%), and pericardial effusion (3\%) (absolute frequencies not available). Thirteen patients (3\%) developed fluid retention that was considered serious and included pericardial effusion $(\mathrm{n}=6 ; 1 \%)$, pleural effusion $(\mathrm{n}=5 ; 1 \%)$, and ascites $(\mathrm{n}=2 ;<1 \%)$. There was also one incident of fatal brain edema. Supraventricular tachyarrhythmias occurred in 25 patients (5\%), while atrial fibrillation was the most commonly observed arrhythmia, occurring in 20 patients (4\%). Other supraventricular tachyarrhythmias that were seen included atrial flutter $(n=4 ; 1 \%)$, supraventricular tachycardia $(n=4 ; 1 \%)$, and atrial tachycardia $(n=1$; $<1 \%$ ). For patients who experienced these supraventricular tachyarrhythmias, 13 (52\%) of the events led to hospitalization. Symptomatic bradyarrhythmias that led to a requirement for pacemaker implantation occurred in three patients (1\%). Treatment-emergent hypertension was common and occurred in 300 patients $(67 \%)$. In patients who were not hypertensive at baseline, nearly half of the patients $(139 / 282 ; 49 \%)$ developed stage 1 hypertension and 82 (29\%) developed stage 2 hypertension. In patients who had stage 1 hypertension at baseline, 80/131 (61\%) developed stage 2 hypertension during ponatinib therapy. Eight (2\%) patients experienced a serious hypertension-related adverse event, such as hypertensive crisis. ${ }^{8}$

Other less common, but important, safety concerns revealed by the PACE trial include the occurrence of pancreatitis, hemorrhage, myelosuppression, tumor lysis syndrome, compromised wound healing, gastrointestinal perforation, and embryo-fetal toxicity. Clinical pancreatitis occurred in 28 patients ( $6 \%$ ). Of these patients, pancreatitis led to discontinuation of therapy in 25 patients ( $89 \%$ ). Most pancreatitis cases (79\%) resolved within 2 weeks following dose interruption or reduction. Hemorrhagic events occurred in $24 \%$ of patients (absolute frequency not available), and the most common events were cerebral hemorrhage and gastrointestinal hemorrhage. Serious hemorrhagic events occurred in $5 \%$ of patients and, included fatalities (absolute number not available). Severe myelosuppression occurred in 215 patients (48\%), and the incidence was similar to that observed in the Phase I trial. Incidence of both hemorrhagic events and myelosuppression was greater in patients with AP-CML, BP-CML, and Ph+ ALL than in patients with CP-CML. Less than $1 \%$ of patients developed serious tumor lysis syndrome, and this was only observed to occur in patients with advanced phases of CML. ${ }^{8}$ Based on its mechanism of action, ponatinib could compromise wound healing and result in fetal harm, however, no formal studies have been conducted on these effects.

\section{Discussion}

Use of TKIs has significantly altered the manner in which CML is treated and has contributed to extending survival and improving outcomes in many patients living with this disease. Despite these advances, therapeutic limitations with existing TKIs created treatment gaps into which a portion of patients fall due to resistance or intolerance. Improved understanding of the atomic interactions of the amino acid structure of BCR-ABL variants has led to the additional design of agents that may overcome these limitations. Ponatinib offers clinicians a potent option that appears to meet the needs of those patients who require alternative TKI therapy. Ponatinib has been associated with the achievement of major cytogenic response and major hematological response in over half of the treated patients with CML in chronic phase, and even provides significant benefit in patients in accelerated phase, blast phase, and $\mathrm{Ph}+\mathrm{ALL}$, despite previous intolerance or refractoriness to other TKIs.

Although the therapeutic efficacy of ponatinib seems promising, patients and their physicians will need to weigh the potential benefits with risks before beginning treatment. Clinicians and patients should be aware of common side effects such as hypertension, rash, abdominal pain, fatigue, headache, dry skin, constipation, fever, joint pain, and nausea. In particular, the cardiovascular safety profile of ponatinib is notable for arterial ischemic events and hypertension. ${ }^{2}$ Other serious adverse events include tumor lysis syndrome, gastrointestinal perforation, and liver toxicity. Due to the risk of significant adverse events, ponatinib's labeling includes a black boxed warning for arterial thrombosis and liver toxicity, which preclude use in certain patient populations and require routine monitoring during treatment.

The ongoing Phase III trial has the potential to show ponatinib utility as first-line therapy in patients with newly diagnosed $\mathrm{CML}$ or $\mathrm{Ph}+\mathrm{ALL}$, and the comparison to imatinib will be invaluable. Ponatinib is currently being evaluated for additional indications such as acute myeloid leukemia and solid tumors. ${ }^{22}$ Ponatinib may also have some utility in treating malignancies that are associated with FGFR1 abnormalities such as myeloid and lymphoid neoplasms associated with FGFR1 (MLNAF) since it targets the FGFR tyrosine kinase 
family in addition to BCR-ABL tyrosine kinases. ${ }^{23}$ Ponatinib has been shown to inhibit in vitro growth of MLNAF cells and has been shown to extend survival in rodent models xenotransplanted with MLNAF cell lines. ${ }^{23}$ Further studies assessing the combination of TKIs by identifying novel targets, mechanisms of actions, or different side effect profiles should be warranted, as this could lead to the discovery of broader types of ABL inhibitors.

\section{Disclosure}

None of the authors have any financial interests to disclose. The authors report no conflicts of interest in this work.

\section{References}

1. Chen Y, Wang H, Hagpo K, Cortes J. Trends in Chronic myeloid leukemia incidence and survival in the United States from 1975-2009. Leuk Lymphoma. 2013;54(7):1411-1417.

2. Gleevec [package insert]. East Hanover, NJ: Novartis Pharmaceuticals Corporation; 2012.

3. Sprycel [package insert]. Princenton, NJ: Bristol-Myers Squibb; 2012.

4. Tasigna [package insert]. East Hanover, NJ: Novartis Pharmaceuticals Corporation; 2012.

5. Jabbour E, Kantarjian H. Chronic myeloid leukemia: 2012 update on diagnosis, monitoring, and management. Am J Hematol. 2012; 87(11):1037-1045.

6. National Cancer Institute [homepage on the Internet]. Chronic Myelogenous Leukemia Treatment. Available from: http://www.cancer.gov. Accessed July 23, 2013.

7. Bosulif [package insert]. New York, NY: Pfizer Labs; 2012.

8. Iclusig [package insert]. Cambridge, MA: Ariad Pharmaceuticals; 2012.

9. National Cancer Institute [homepage on the Internet]. Childhood Acute Lymphoblastic Leukemia Treatment. Available from: http://www.cancer. gov. Accessed July 23, 2013.

10. National Cancer Institute [homepage on the Internet]. Adult Acute Lymphoblastic Leukemia Treatment. Available from: http://www.cancer. gov. Accessed July 23, 2013.

11. Hoelzer D, Thiel E, Löffler H, et al. Prognostic factors in a multicenter study for treatment of acute lymphoblastic leukemia in adults. Blood. 1988;71(1):123-131.
12. Gaynor J, Chapman D, Little C, et al. A cause-specific hazard rate analysis of prognostic factors among 199 adults with acute lymphoblastic leukemia: the Memorial Hospital experience since 1969. J Clin Oncol. 1988;6(6):1014-1030.

13. Koo HH. Philadelphia chromosome-positive acute lymphoblastic leukemia in childhood. Korean J Pediatr. 2011;54(3):106-110.

14. Zuccotto F, Ardini E, Casale E, Angiolini M. Through the "gatekeeper door": exploiting the active kinase conformation. J Med Chem. 2010;53(7):2681-2694.

15. Zhou T, Commodore L, Huang WS, et al. Structural Mechanism of the Pan-BCR-ABL inhibitor ponatinib (AP24534): lessons for overcoming kinase inhibitor resistance. Chem Biol Drug Des. 2011;77(1):1-11.

16. O'Hare T, Shakespeare WC, Zhu XT, et al. AP24534, a pan-BCRABL inhibitor for chronic myeloid leukemia, potently inhibits the T315I mutant and overcomes mutation-based resistance. Cancer Cell. 2009; $16: 5$ (401-412).

17. Cortes JE, Kantarjian H, Shah NP, et al. Ponatinib in refractory Philadelphia chromosome-positive leukemias. $N$ Engl J Med. 2012; 367(22):2075-2088.

18. US Food and Drug Administration, Center for Drug Evaluation and Research Medical Review Iclusig ${ }^{\circledR}$ NDA 203469 Application. Available December 21, 2012. Silver Spring, MD. Available from: http://www. accessdata.fda.gov/drugsatfda_docs/nda/2012/203469Orig1s000MedR. pdf. Accessed January 16, 2013.

19. ARIAD Pharmaceuticals. ARIAD announces initiation of randomized Phase 3 trial of Ponatinib in newly diagnosed patients with chronic myeloid leukemia [press release]. Cambridge, MA: ARIAD Pharmaceuticals; [July 27, 2012]. Available from: http://phx.corporate-ir.net/phoenix.zhtml?c=118422\&p=irol-newsArticle \&ID=1719432\&highlight=. Accessed December 19, 2012.

20. National Cancer Institute. Common Terminology Criteria for Adverse Events v3.0. Bethesda, MD: National Cancer Institute; 2006. Available from: http://ctep.cancer.gov/protocolDevelopment/electronic_ applications/docs/ctcaev3.pdf. Accessed April 9, 2013.

21. US Food and Drug Administration, Center for Drug Evaluation and Research, Office Director Decisional Memo 203469 Iclusig $^{\circledR}$ (ponatinib). Silver Spring, MD. Available from: http://www.accessdata. fda.gov/drugsatfda_docs/nda/2012/203469Orig1s000ODMemo.pdf. Accessed April 9, 2013.

22. Ariad Pharmacetuicals. Ariad Pipeline. Available from: http://www. ariad.com/pipeline. Accessed April 24, 2013.

23. Ren M, Qin H, Ren R, Cowell JK. Ponatinib suppresses the development of myeloid and lymphoid malignanies associated with FGFR1 abnormalities. Leukemia. 2013;27(1):32-40.
OncoTargets and Therapy

\section{Publish your work in this journal}

OncoTargets and Therapy is an international, peer-reviewed, open access journal focusing on the pathological basis of all cancers, potential targets for therapy and treatment protocols employed to improve the management of cancer patients. The journal also focuses on the impact of management programs and new therapeutic agents and protocols on

\section{Dovepress}

patient perspectives such as quality of life, adherence and satisfaction. The manuscript management system is completely online and includes a very quick and fair peer-review system, which is all easy to use. Visit http://www.dovepress.com/testimonials.php to read real quotes from published authors. 